\title{
FK506-binding protein 51 (FKBP5) gene polymorphism is not associated with glucocorticoid therapy outcome in patients with idiopathic thrombocytopenic purpura
}

\author{
WANSHENG LAO ${ }^{1}$, MEIYUN FANG ${ }^{1}$ and XIFEI YANG ${ }^{2}$ \\ ${ }^{1}$ Department of Hematology, The First Affiliated Hospital of Dalian Medical University, Dalian, Liaonin 116011; \\ ${ }^{2}$ Key Laboratory of Modern Toxicology of Shenzhen, Shenzhen Center for \\ Disease Control and Prevention, Shenzhen 518055, P.R. China
}

Received March 2, 2012; Accepted July 9, 2012

DOI: $10.3892 / \mathrm{mmr} .2012 .993$

\begin{abstract}
The FKBP5 gene codes for the FK506-binding protein 51 (FKBP5), a co-chaperone of hsp90, which regulates glucocorticoid (GC) receptor sensitivity. The FKBP5 gene single nucleotide polymorphisms (SNP), rs1360780, has been found to modulate GC sensitivity in stress-related psychiatric disorders. The aim of the present study was to examine the effects of rs1360780 on the treatment outcome of patients suffering from idiopathic thrombocytopenic purpura (ITP) administered with GC. The polymorphism of FKBP5, rs1360780, was genotyped in 55 GC-resistant ITP patients, 157 GC-sensitive ITP patients and 110 unrelated healthy individuals using real-time PCR and cycling probe technology with DNA extracted from peripheral blood. No significant differences in FKBP5 rs1360780 genotypes $(\mathrm{P}=0.51)$ and alleles $(\mathrm{P}=0.89)$ were observed between the GC-resistant ITP patients and the healthy controls. There were no significant differences observed between the GC-sensitive ITP patients and the healthy controls $(\mathrm{P}=0.40$ for genotypes and $\mathrm{P}=0.62$ for $\mathrm{T}$ allele), as well as between the $\mathrm{GC}$-sensitive ITP patients and the GC-resistant patients $(\mathrm{P}=0.67$ for genotypes and for $\mathrm{T}$ allele). The present study demonstrates that the FKBP5 polymorphism may not affect the response of ITP patients to GC treatment.
\end{abstract}

\section{Introduction}

Idiopathic thrombocytopenic purpura (ITP) is considered to be an organ-specific autoimmune disorder characterized by a severe decrease in platelet numbers and mucocutaneous bleeding (1). ITP-associated platelets are opsonized by autoantibodies and prematurely destroyed by the reticuloendothelial

Correspondence to: Dr Meiyun Fang, Department of Hematology, The First Affiliated Hospital of Dalian Medical University, 222 Zhongshan Road, Dalian, Liaonin 116011, P.R. China

E-mail: fangmeiyun@yahoo.com.cn

Key words: idiopathic thrombocytopenic purpura, glucocorticoid resistance, FK506-binding protein 51 system (2-4). The incidence of ITP is estimated to be 6 cases per 100,000 individuals per year in Western developed countries (5). Glucocorticoids (GCs) in combination with romiplostim are currently the mainstay of ITP therapy (6-7). Generally, the majority of patients with ITP respond favorably to GCs and never experience serious bleeding even with severe thrombocytopenia. However, a fraction of patients with ITP develop GC resistance following 3-6 months of standard treatment with GC (8). The mechanisms by which GC resistance develops are not yet fully understood. Three possible mechanisms that could to lead to the development of GC resistance have been proposed: reduced numbers of glucocorticoid receptors (GRs), altered affinity of the ligand for GRs and reduced ability of the GRs to bind the DNA (9).

The FKBP5 gene is located on the short arm in human chromosome 6 and consists of 10 exons (10). The FKBP5 gene codes for FK506-binding protein 51 (FKBP5), a co-chaperone of hsp90, which regulates GR sensitivity (11). Functionally, FKBP5 expression is induced by steroids which activate GR elements (12). In turn, in vitro experiments have demonstrated that FKBP5 reduces the hormone binding affinity and decreases the amount of activated GR translocation to the cell nucleus (13).

FKBP5 is recognized to be expressed as several polymorphic variants, but the FKBP5 gene single nucleotide polymorphism (SNP), rs1360780, has been shown to modulate GC sensitivity in stress-related psychiatric disorders and has been associated with altered response to antidepressant drugs $(14,15)$. FKBP51 mRNA expression in the peripheral blood has been used to determine individual sensitivity to corticosteroids in an in vitro bioassay (12). In addition, Woodruff et al identified FKBP51 as a biomarker of GC responsiveness and a potential mediator of GC-resistant asthma (16). However, it remains unknown whether rs1360780 may influence FKBP5 protein levels to develop GC resistance in ITP. The aim of the present study was to examine the effects of rs1360780 on the treatment outcome in patients suffering from ITP administered with GC.

\section{Subjects and methods}

Subjects and study design. A total of 212 Chinese patients with ITP and 110 unrelated healthy individuals were recruited from 
Table I. Clinical characteristics of ITP subjects.

\begin{tabular}{lccc}
\hline & \multicolumn{2}{c}{ Group } & Control \\
\cline { 2 - 4 } Clinical characteristics & ITP (GC-R) & ITP (GC-S) & 110 \\
\hline Number of subjects & 55 & 157 & $43: 67$ \\
Gender (male:female) & $23: 32$ & $53: 104$ & $41(19-70)$ \\
Age at study entry, median (range in years) & $37(20-74)$ & $40(18-75)$ & \\
Disease course, median (range in years) & $1.2(0.3-37)$ & $1.0(0.1-38)$ & \\
\hline
\end{tabular}

ITP, idiopathic thrombocytopenic purpura; GC-S, glucocorticoid-sensitive; GC-R, glucocorticoid-resistant.

Table II. Distribution and statistical analysis of rs1360780 allelic and genotypic frequencies in control and ITP patients.

\begin{tabular}{|c|c|c|c|c|c|}
\hline \multirow[b]{2}{*}{ FKBP5 rs1360780 } & \multicolumn{3}{|c|}{ Frequency } & \multirow[b]{2}{*}{ P-value } & \multirow{2}{*}{$\begin{array}{l}\text { Odds ratio } \\
(95 \% \text { CIs) }\end{array}$} \\
\hline & $\operatorname{ITP}(\mathrm{GC}-\mathrm{R})^{\mathrm{a}}$ & Control $^{\mathrm{b}}$ & $\operatorname{ITP}(\mathrm{GC}-\mathrm{S})^{\mathrm{c}}$ & & \\
\hline Number of subjects & 55 & 110 & 157 & & \\
\hline \multicolumn{6}{|l|}{ Allele } \\
\hline $\mathrm{C}$ & $86(0.78)$ & $169(0.77)$ & $234(0.75)$ & $0.89^{\mathrm{a}}$ & $0.92^{\mathrm{a}}(0.53-1.60)$ \\
\hline $\mathrm{T}$ & $24(0.22)$ & $51(0.23)$ & $80(0.25)$ & $\begin{array}{l}0.62^{\mathrm{b}} \\
0.52^{\mathrm{c}}\end{array}$ & $\begin{array}{l}1.13^{\mathrm{b}}(0.76-1.70) \\
1.23^{\mathrm{c}}(0.73-2.06)\end{array}$ \\
\hline \multicolumn{6}{|l|}{ Genotype } \\
\hline $\mathrm{CC}$ & $32(0.59)$ & $65(0.59)$ & $83(0.53)$ & $0.51^{\mathrm{a}}$ & \\
\hline CT & $22(0.39)$ & $39(0.36)$ & $68(0.43)$ & $0.40^{\mathrm{b}}$ & \\
\hline $\mathrm{TT}$ & $1(0.02)$ & $6(0.05)$ & $6(0.04)$ & $0.67^{\mathrm{c}}$ & \\
\hline
\end{tabular}

${ }^{a}$ ITP (GC-R) vs. healthy controls; ${ }^{\mathrm{b}} \mathrm{ITP}$ (GC-S) vs. healthy controls; ' ITP (GC-S) vs. ITP (GC-R). CI, confidence intervals; ITP, idiopathic thrombocytopenic purpura; GC-S, glucocorticoid-sensitive; GC-R, glucocorticoid-resistant.

The First Affiliated Hospital of Dalian Medical University, Dalian, China, between January 2009 and December 2011. The patients were treated with GC and classified into 2 groups: GC-resistant and GC-sensitive (Table I). Among the patients, 55 patients were classified as GC-resistant, while 157 patients were classified as GC-sensitive. The criteria for the outcome of GC therapy (success or failure) was based on the practice guidelines of the American Society of Hematology (2) and guidelines by the General Haematology Task Force of the British Committee for Standards in Haematology (5). The Research Ethics Committee approved the study. After obtaining informed consent, all patients and controls were recruited for clinical assessment and collection of blood samples for extraction of genomic DNA.

SNP selection and genotyping. DNA was extracted from the peripheral blood using a blood extraction kit (Takara Bio, Inc., Shiga, Japan) according to the manufacturer's instructions. Real-time PCR and cycling probe technology (Takara) was used to detect the FKBP5 rs1360780 SNP. The primer design was based on published sequences for genotyping the FKBP5 polymorphism using genomic DNA. The primers and probes were as follows: 5'-AGC ACA TAA TAG CAA TTC ACA-3' (forward primer), 5'-TGG CTT TAG TCA CAT TAA ATA G-3' (reverse primer), 5'(Eclipse)-CAA AGT TAC ACA-(FAM)3', and 5'(Eclipse)-ATA CAA AAC AAA A-(ROX)3', where underlined characters indicate the RNA (17). The PCR amplification and fluorescence detection were performed using a Roche Light Cycler 480 (Roche Bio Inc., Rotkreuz, Switzerland). Cycle conditions were as follows; holding at $95^{\circ} \mathrm{C}$ for $30 \mathrm{sec}, 45$ cycles of denaturation at $95^{\circ} \mathrm{C}$ for $5 \mathrm{sec}$, primer annealing at $55^{\circ} \mathrm{C}$ for $10 \mathrm{sec}$, and elongation at $72^{\circ} \mathrm{C}$ for $20 \mathrm{sec}$. Fluorescence was measured at $72^{\circ} \mathrm{C}$.

Statistical analysis. SPSS 13.0 was used for statistical analysis. Allelic and genotypic frequencies were analyzed using the Chi-square test or Fisher's exact test in case of expected frequencies $<5$. Odds ratios (ORs) and $95 \%$ confidence intervals (CIs) and were also calculated. $\mathrm{P}<0.05$ was considered to indicate a statistically significant difference.

\section{Results}

The distribution of CC, CT and TT genotypes was 59, 39 and $2 \%$, respectively. The allelic frequencies of $\mathrm{C}$ and $\mathrm{T}$ in the GC-resistant ITP patients were 78 and $22 \%$, respectively (Table II). The distribution of CC, CT and TT genotypes was 59,36 and $5 \%$, respectively. The allelic frequencies of $\mathrm{C}$ 
and $\mathrm{T}$ in the healthy control patients were 77 and $23 \%$, respectively. No significant differences were observed in FKBP5 rs1360780 genotypes $(\mathrm{P}=0.51)$ and alleles $(\mathrm{P}=0.89$; $\mathrm{OR}, 0.92$; CI, 0.53-1.60) between the GC-resistant ITP patients and the healthy controls. The distribution of CC, CT and TT genotypes was 53, 43 and 4\%, respectively. The allelic frequencies of $\mathrm{C}$ and $\mathrm{T}$ in the GC-sensitive ITP patients were 75 and $25 \%$, respectively. There were no significant differences observed between the GC-sensitive ITP patients and the healthy controls $(\mathrm{P}=0.40$ for genotypes and $\mathrm{P}=0.62$; OR, 1.13; $\mathrm{CI}, 0.76-1.70$ for $\mathrm{T}$ allele), as well as between the GC-sensitive ITP patients and the $\mathrm{GC}$-resistant patients $(\mathrm{P}=0.67$ for genotypes and $\mathrm{P}=0.52$, OR, 1.23; CI, 0734-2.06 for T allele).

\section{Discussion}

GCs are currently one of the most widely used anti-inflammatory agents. SNPs in the FKBP5 gene affecting the sensitivity of GC have been investigated in stress-related psychiatric disorders, asthma, Crohn's disease and ulcerative colitis $(14,16,18)$; however, no studies have examined the correlation between FKBP5 polymorphisms and GC efficacy in Chinese cases with ITP. In the present study, we evaluated the effects of the FKBP5 polymorphism, rs1360780, on the efficacy of GC treatment for ITP cases. We observed no significant differences in FKBP5 rs1360780 genotypes and alleles between the GC-resistant ITP patients and the healthy controls, between the GC-sensitive ITP patients and the healthy controls, as well as between the GC-sensitive ITP patients and the GC-resistant patients. Our results demonstrate that the rs1360780 SNP of FKBP5 may not be associated with GC treatment response.

Since FKBP5 protein expression may have an impact on cellular sensitivity to systemic GC treatment (19), abnormalities in FKBP5 SNPs may contribute to decreased GC responsiveness. Previous studies have suggested that FKBP5 polymorphisms are associated with GC sensitivity. Hubler and Scammell reported that polymorphisms of the FKBP51 gene (rs1360780) are linked with an increased FKBP5 protein expression; the GR regulatory region of FKBP5 mRNA is located in the region $<200$ bp away from the highly conserved and functional hormone response element in intron 2 of the gene (20). Ising et al showed that compared to the patients carrying the other alleles, the highinduction alleles of FKBP5 were associated with GR resistance in healthy controls and enhanced GR sensitivity in depressed patients (21). In addition, the rs1360780 $\mathrm{T}$ allele was associated with an increased cortisol response as demonstrated by investigating the cortisol reactivity of 14-month-old infants during a stress test (22). These data suggest that FKBP5 polymorphisms affect GC sensitivity. However, similar to our results, other studies have demonstrated an association between FKBP5 polymorphisms and GC sensitivity. Sarginson et al duplicated findings by Binder et al (14) in a cohort of 246 elderly patients who suffered major depression treated with either mirtazapine or paroxetine, but could not identify polymorphisms in FKBP5 gene affecting GR sensitivity (23). Furthermore, Papiol et al did not identify any association between FKBP5 genotypes and GC sensitivity in a Spanish study using citalopram (24). In addition, Maltese et al reported that the rs1360780 SNP of FKBP5 was not associated with glucocorticoid resistance in Crohn's disease and ulcerative colitis (18).
These inconsistences may arise from the environmental and ethnic differences between the populations in the collected samples. Although the data concerning polymorphisms of FKBP5 gene influence on GC sensitivity are so far inconclusive and controversial, by comparing previous studies the present study provides further evidence supporting the close correlation between FKBP5 polymorphism and GC sensitivity.

In conclusion, this is the first attempt to correlate the response to GC with FKBP5 gene polymorphisms in ITP patients. However, several main limitations should also be considered in this study. Firstly, due to the limited availability of patient samples, our study included relatively small sample sizes. Larger case-control studies are required to further assess the role of the FKBP5 rs1360780 polymorphism and GC therapy in ITP patients. Secondly, selection of the SNP was based on FKBP5 rs1360780; thus, we cannot fully exclude the possibility that other SNPs located somewhere within the gene locus are also associated with GC therapy. Nevertheless, the present study demonstrates that the FKBP5 polymorphism does not affect the response of ITP patients to GC treatment.

\section{Acknowledgements}

The authors thank the patients for the blood donation and agreement to participate in this study. This study was supported by the Key Clinical Research Project of Public Health Ministry of China, Commonweal Trade for Scientific Research (200802031), the Clinical Research Project of Chinese Medical Doctor Association (20100136) and the Scientific Research Project of Dalian City Committee of Science and Technology (2009E12SF166).

\section{References}

1. Zhou ZP and Yang RC: Rituximab treatment for chronic refractory idiopathic thrombocytopenic purpura. Hematology 65 : 21-31, 2008.

2. George JN, Woolf SH and Raskob GE: ITP: practice guideline developed by explicit methods for the American Society of Hematology. Blood 88: 3-40, 1996.

3. Beardsley DS and Ertem M: Platelet autoantibodies in immune thrombocytopenic purpura. Transfus Sci 9: 237-244, 1998.

4. Kuwana M, Okazaki Y, Kaburaki J and Ikeda Y: Detection of circulating B cells secreting platelet-specific autoantibody is useful in the diagnosis of autoimmune thrombocytopenia. Am J Med 114: 322-325, 2003.

5. British Committee for Standards in Haematology: General Haematology Task Force: Guidelines for the investigation and management of idiopathic thrombocytopenic purpura in adults, children and in pregnancy. Br J Haematol 120: 574-596, 2003.

6. Cines DB and McMillan R: Management of adult idiopathic thrombocytopenic purpura. Annu Rev Med 56: 425-442, 2005.

7. Meletis J, Katsandris A, Raptis S and Mantzourani M: Successful treatment of immune thrombocytopenic purpura (ITP) with the thrombopoietin-mimetic romiplostim. Med Sci Monit 16: 100-102, 2010.

8. Kojouri and George JN: Recent advances in the treatment of chronic refractory immune thrombocytopenic purpura. Int $\mathbf{J}$ Hematol 81: 119-125, 2005.

9. Pierik M, Rutgeerts $P$, Vlietinck $R$ and Vermeire S: Pharmacogenetics in inflammatory bowel disease. World $\mathrm{J}$ Gastroenterol 12: 3657-3667, 2006.

10. Nair SC, Rimerman RA, Toran EJ, Chen S, Prapapanich V and Butts RN: Molecular cloning of human FKBP51 and comparisons of immunophilin interactions with Hsp90 and progesterone receptor. Mol Cell Biol 17: 594-603, 1997.

11. Binder EB: The role of FKBP5, a co-chaperone of the glucocorticoid receptor in the pathogenesis and therapy of affective and anxiety disorders. Psychoneuroendocrinology 34: 186-195, 2009. 
12. Vermeer H, Hendricks-Stegemann BAI, Van der Burg B, van Buul Offers SC, and Jansen M: Glucocorticoid-induced increase in lymphocytic FKBP5 messenger ribonucleic acid expression: a potential marker for glucocorticoid sensitivity, potency, and bioavailability. J Clin Endocrin Metabol 88: 277-284, 2003.

13. Wochnik GM, Ruegg J, Abel GA, Schmidt U, Holsboer F and Rein T: FK506-binding proteins 51 and 52 differentially regulate dynein interaction and nuclear translocation of the glucocorticoid receptor in mammalian cells. J Biol Chem 28: 4609-4616, 2005.

14. Binder EB, Salyakina D, Lichtner P, Wochnik GM, Ising M and Putz B, et al: Polymorphisms in FKBP5 are associated with increased recurrence of depressive episodes and rapid response to antidepressant treatment. Nat Gen 36: 1319-1325, 2004.

15. Tatro ET, Everall IP, Masliah E, Hult BJ, Lucero G and Chana G: Differential expression of immunophilins FKBP51 and FKBP52 in the frontal cortex of HIV-infected patients with major depressive disorder. J Neuroimmune Pharmacol 4: 218-226, 2009.

16. Woodruff PG, Boushey HA, Dolganov GM, Barker CS, Yang YH, Donnelly $\mathrm{S}$, et al: Genome-wide profiling identifies epithelial cell genes associated with asthma and with treatment response to corticosteroids. Proc Natl Acad Sci USA 104: 15858-15863, 2007.

17. Shibuya N, Suzuki A, Sadahiro R, Kamata M, Matsumoto Y, Goto K, Hozumi Y and Otani K: Association study between a functional polymorphism of FK506-binding protein 51 (FKBP5) gene and personality traits in healthy subjects. Neurosci Lett 485: 194-197, 2010.

18. Maltese P, Palma L, Sfara C, Rocco PD, Latiano A, Palmieri O, Corritore G, Annese V and Magnani M: Glucocorticoid resistance in Crohn's disease and ulcerative colitis: an association study investigating GR and FKBP5 gene polymorphisms. Pharmacogenomics 26: 1-7, 2011.
19. Scammell JG, Denny WB, Valentine DL and Smith DF: Overexpression of the FK506-binding immunophilin FKBP51 is the common cause of glucocorticord resistance in three New World primates. Gen Comp Endocrinol 124: 152-165, 2001.

20. Hubler TR and Scammell JG: Intronic hormone response elements mediate regulation of FKBP5 by progestins and glucocorticoids. Cell Stress Chaperones 9: 243-252, 2004.

21. Ising M, Depping AM, Siebertz A, Lucae S, Unschuld PG, Kloibers S, Horstmann S, Uhr M, Müller-Myhsok B and Holsboer F: Polymorphisms in the FKBP5 gene region modulate recovery from psychosocial stress in healthy controls. Eur J Neurosci 28: 389-398, 2008.

22. Luijk MP, Velders FP, Tharner A, van Ijzendoorn MH, Bakermans-Kranenburg MJ, Jaddoe VW, Hofman A, Verhulst FC and Tiemeieret H: FKBP5 and resistant attachment predict cortisol reactivity in infants: gene-environment interaction. Psychoneuroendocrinology 35: 1454-1461, 2010.

23. Sarginson JE, Lazzeroni LC, Ryan HS, Schatzberg AF and Murphy GM Jr: FKBP5 polymorphisms and antidepressant response in geriatric depression. Am J Med Genet B Neuropsychiatr Genet 153: 554-560, 2010.

24. Papiol S, Arias B, Gasto C, Gutierrez B, Catalan R and Fananas L: Genetic variability at HPA axis in major depression and clinical response to antidepressant treatment. J Affect Disord 104: 83-90, 2007. 